\title{
Comparing Parameter Estimates Obtained by Simulation Study and Real Life Data from the Two-Parameter Gamma Model
}

\author{
A. M. Yahaya, N. P. Dibal, H. R. Bakari \\ Department of Mathematics and Statistics, University of Maiduguri, Maiduguri, Nigeria \\ Email address: \\ abdullstat@yahoo.com (A. M Yahaya)
}

\section{To cite this article:}

A. M. Yahaya, N. P. Dibal, H. R. Bakari. Comparing Parameter Estimates Obtained by Simulation Study and Real Life Data from the TwoParameter Gamma Model. International Journal of Statistical Distributions and Applications. Vol. 3, No. 2, 2017, pp. 13-17. doi: $10.11648 /$ j.ijsd.20170302.11

Received: August 27, 2016; Accepted: November 30, 2016; Published: May 6, 2017

\begin{abstract}
The aim of this study was to employ Maximum Likelihood (MLE) jointly with a numerical Method (Newton Raphson method) to obtain parameter estimates from the two-parameter Gamma model. The profile likelihood of the twoparameter Gamma model was also put into consideration. The methods were demonstrated using simulation studies and real life data considering data sets generated by R statistical software for different sample sizes. Standard errors were computed and $5 \%$ Wald-confidence interval was constructed for the estimates of the model. The result of the study shows that Maximum Likelihood Estimation (MLE) jointly with Newton Raphson method was more efficient for estimating parameters of the Gamma model in simulation study than real life data. The study recommends that parameter estimates from the two-parameter Gamma model should be obtained by employing Maximum Likelihood Estimation jointly with Newton Raphson Method.
\end{abstract}

Keywords: Parameter Estimation, Two-Parameter Gamma Model, Profile Likelihood, Maximum Likelihood Estimation, Newton Raphson Method

\section{Introduction}

The two-parameter gamma probability distribution has found much application in technology and natural sciences, more especially in the areas of failure and survival analysis. The estimation of the parameters of the two-parameter gamma distribution is of great importance and it has been discussed widely. The maximum likelihood (MLE) is the most commonly used method of parameter estimation due its efficiency and good theoretical properties, and its ease application which gives explicit algebraic estimates of a probability distribution. However, in some cases, the MLE is ineffective in terms of statistical or computation properties, in particular for Gamma Probability distribution. The maximum likelihood (MLE) does not provide explicit estimates of unknown parameters in Gamma model and require the application of other numerical methods. Therefore, other methods which have been proposed in the literature as an alternative to the MLE method include; the secant, the bisection and the Newton-Raphson Methods. However, in both the secant and the bisection methods, the process converges slowly. The Newton-Raphson method on the other hand computes both the basic estimating function and its derivative at each iterative step and it converges very fast. Furthermore, the MLE-based methods require either initial values or trial computation for the estimation of the parameters. In this study, MLE was used jointly with the Newton Raphson method which has the ability to find solutions where no closed form exist, ease of application and faster rate of convergence for the estimation of the parameters of two-parameter Gamma model using simulation studies and real life data.

\section{Review of Related Literature}

The likelihood function tells us how likely the observed sample is a function of the possible parameter values. Thus, maximizing the likelihood function for the data gives the parameter values for which the observed sample is most likely to have been generated, that is, the parameter values 
that "agree most closely" with the observed data (Fisher,1920).Modern applied statistics deals with many settings in which the point wise evaluation of the likelihood function is impossible or computationally difficult there by making it difficult to perform any inference (classical or Bayesian) about the parameters of the model. Areas such as financial modelling, genetics, geostatistics, neurophysiology and stochastic dynamical systems provides numerous example of this (Pritchard et al., 1999).Various approaches to overcome the shortcomings of MLE has been proposed by several researchers; Cox and Reid (2004) used Composite Likelihood methods for approximating the likelihood function, also Pritchard et al., 1999 and Beaumont et al., 2002, applied the Approximate Bayesian Computational methods for approximating the posterior distribution to obtain the estimates of parameters. It is well-known that $\mathrm{ABC}$ produces a sample approximation of the posterior distribution in which there exist a deterministic approximation error in addition to Monte Carlo variability (Beaumont et al., 2002). The posterior and theoretical properties of the estimators when the $\mathrm{ABC}$ methods is applied and its use in model comparison have been studied and presented in many works (Wilkinson, 2008; Didelot et al., 2011; Robert et al., 2011; Marin et al., 2011; Dean et al., 2011; Fearnhead and Prangle, 2012).Using the sample approximation to characterize the mode of the posterior would in principle allow (approximate) maximum a posteriori (MAP) estimation. Furthermore, using a uniform prior distribution, under the parameters of interest over any set which contains the MLE will lead to a MAP estimate which coincides with the MLE. In low-dimensional problems if we have a sample from the posterior distribution of the parameters, we can estimate its mode by using either nonparametric estimators of the density or another mode seeking technique such as the mean-shift algorithm (Fukunaga and Hostetler, 1975). Although Marjoram et al., (2003) noted that $(\mathrm{ABC})$ can also be used in frequentist applications, more especially in for maximum-likelihood estimation; this approach did not receive much attention. Alternative nonparametric density estimators which could also be considered within the AMLE context have been proposed recently (Culeet al., 2010; Jing et al., 2012). Cheng and Amin (1983) suggested the maximum product of spacing (MPS) method which can be applied to any univariate distribution. Cheng and Traylor (1995) pointed out the drawbacks of the MPS method owing to the occurrence of tied observations and numerical effects involved in ordering the cumulative density function when there are explanatory variables in the model. Atkinson and Pericchiet al., (1991) applied the grouped-data likelihood approach to the shifted power transformation model of Box and Cox (1964).

\section{The Gamma Probability Distribution}

In probability theory and statistics, Gamma distribution is a member of two-parameter family of continuous probability distributions. It has a scale parameter $\alpha$ and a shape parameter $\lambda$. If $\alpha$ is an integer then the distribution represents the sum of $\alpha$ independent exponentially distributed random variables, each of which has a mean of $\lambda$ (which is equivalent to a rate parameter of $\lambda-1$ ). The gamma distribution is frequently a probability model for waiting times; for instance, in life testing, the waiting time until death is a random variable that is frequently modeled with a gamma distribution.

\subsection{Materials and Methods}

The study employed MLE jointly with Numerical method (Newton-Raphson method) to obtain the estimates, profilelikelihood, standard errors and Wald interval of the twoparameter Gamma model using simulation studies and real life data which was implemented using R Statistical software.

\subsection{The Maximum Likelihood Estimation Method}

The maximum likelihood estimation method had been used in special cases by Gauss in 1812 but a full description of properties and a presentation of its application were performed 100 years later by Ronald Fisher [1]. Nowadays, the maximum likelihood method is the most popular estimation technique, mainly for its good theoretical properties. See other literature [7, 14, 17, 19] for the existence and the uniqueness of the maximum likelihood estimates for discussed distributions. The idea of the maximum likelihood method is based on the assumption that observed data are the most likely outcome of a random experiment in respect to the considered probability distribution. In the discussed method, the key role plays the likelihood function specified as the probability of observed data depending on the values of distribution parameters.

Table 1. Gamma Probability Distribution Mass Function.

\begin{tabular}{ll}
\hline Distribution & $\boldsymbol{f}(\boldsymbol{x}, \boldsymbol{\lambda}, \boldsymbol{k})$ \\
\hline Gamma & $x^{k-1} \cdot \frac{e^{-\frac{x}{\lambda}}}{\Gamma(k) \cdot \lambda^{k}}, x \geq 0, \lambda>0, k>0$ \\
\hline
\end{tabular}

In the above expressions $\lambda$ and $k$ denotes the scale of the shape distribution parameters

Table 2. Gamma Probability Distribution Likelihood Function L.

\begin{tabular}{ll}
\hline Distribution & $\boldsymbol{L}\left(\boldsymbol{\lambda}, \boldsymbol{k}, \boldsymbol{x}_{\mathbf{1}}, \ldots, \boldsymbol{x}_{\boldsymbol{n}}\right)$ \\
\hline Gamma & $\frac{1}{\Gamma(k) \cdot \lambda^{n}} \prod_{i=1}^{n}\left(\frac{x_{i}}{\lambda}\right)^{k-1} \cdot e^{-\sum_{i=1}^{n} \frac{x_{i}}{\lambda}}$ \\
\hline
\end{tabular}

The maximum likelihood estimators of the distribution parameters are found by maximizing the likelihood functions L (actually it logarithms) with respect to parameter values. Maximum likelihood estimates of the shapes and scale parameters for the Gamma distribution is therefore the solution of equations which are obtained by equating partial derivatives of $\operatorname{In}(\mathrm{L})$ to zero presented in Table 3 
Table 3. Gamma Distribution Maximum Likelihood Estimates.

\begin{tabular}{ll}
\hline Distribution & ML Eestimates \\
\hline Gamma & $\bar{x}=\hat{k} \cdot \sqrt[n]{\prod_{i=1}^{n} x_{i} \cdot \exp \left(-\frac{\Gamma \cdot(\hat{k})}{\Gamma(\hat{k})}\right)}, \hat{\lambda}=\frac{\bar{x}}{k}$ \\
\hline
\end{tabular}

The discussed problems have no explicit algebraic solutions; therefore numerical calculations are required in the real life data and simulation studies for the Maximum likelihood estimates using Newton-Raphson's method.

\subsection{Numerical Method (Simulation Study) Gamma Model}

Table 4. Gamma Model Result.

\begin{tabular}{llllll}
\hline $\mathbf{N}$ & $\widehat{\boldsymbol{\alpha}} \boldsymbol{m} \boldsymbol{l} \boldsymbol{e}$ & $\hat{\boldsymbol{\lambda}} \boldsymbol{m} \boldsymbol{l} \boldsymbol{e}$ & $\mathbf{S . E}(\hat{\boldsymbol{\alpha}})$ & S.E $(\hat{\boldsymbol{\lambda}})$ & Wald C.I for $\hat{\boldsymbol{\alpha}}$ \\
\hline 10 & 2.728430 & 9.921558 & 1.154090 & 4.607174 & $1.574339,7.335604$ \\
100 & 2.728430 & 9.921558 & 0.3649554 & 1.4569164 & $2.363474,4.185346$ \\
1000 & 2.728428 & 9.921553 & 0.1154090 & 0.4607172 & $2.3613019,1.89145$ \\
10000 & 2.728428 & 9.921553 & 0.03649552 & 0.14569157 & $2.691933,2.874120$ \\
\hline
\end{tabular}

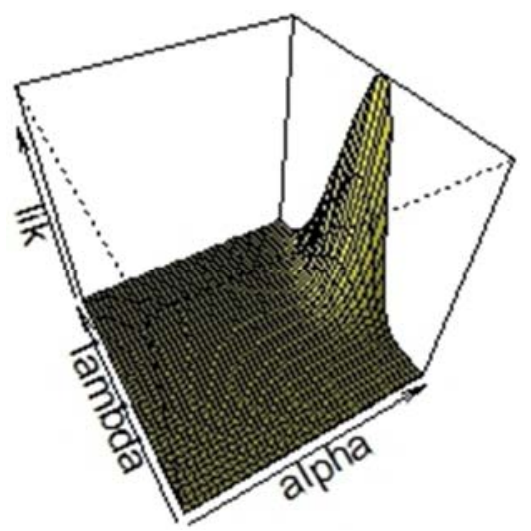

Figure 1. Loglikelihood graph of a Gamma Model.

\subsection{Profile Likelihood of Gamma Distribution}

Table 5. Profile Log - Likelihood of a Gamma Model Result Table.

\begin{tabular}{llll}
\hline $\mathbf{N}$ & $\hat{\lambda} \boldsymbol{m} \boldsymbol{l} \boldsymbol{e}$ & S.E $(\hat{\boldsymbol{\lambda}})$ & Wald C.I for $\hat{\boldsymbol{\lambda}}$ \\
\hline 10 & 9.921523 & 0.7082003 & $2.463034,3.633315$ \\
100 & 9.921535 & 0.2237425 & $4.628043,5.075528$ \\
1000 & 9.921536 & 0.07187667 & $4.954930,5.098683$ \\
10000 & 9.921537 & 0.02275701 & $5.017715,5.063229$ \\
\hline
\end{tabular}

\subsection{The Numerical Method (Real Life Data) Gamma Model}

Table 6. Gamma Model Result Table.

\begin{tabular}{llllll}
\hline$\hat{\alpha} \boldsymbol{m}$ le & $\boldsymbol{S} . \boldsymbol{E}(\hat{\boldsymbol{\alpha}})$ & Wald CI & $\hat{\boldsymbol{\lambda}} \boldsymbol{m l e}$ & S. $\boldsymbol{E}(\hat{\boldsymbol{\lambda}})$ & Wald CI \\
\hline 0.851554121 & 0.1141584591 & $0.7373957,008518967$ & 0.001817164 & 0.0003426042 & $-0.112341295,0.002159768$ \\
\hline
\end{tabular}

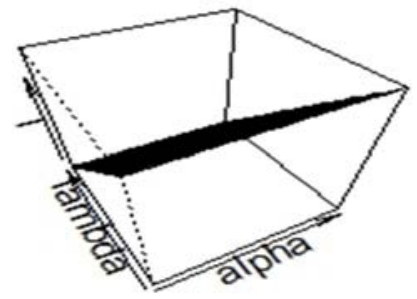

Figure 3. Loglikelihood graph of a Gamma Model.

\subsection{Profile Likelihood of Gamma Model}

Table 7. Profile Likelihood of Gamma Model Result Table.

\begin{tabular}{lll}
\hline$\hat{\propto} \boldsymbol{m l e}$ & $\boldsymbol{S} . \boldsymbol{E}(\hat{\propto})$ & Wald CI \\
1.9484034 & 0.01655228 & $1.967482,2.000587$ \\
\hline
\end{tabular}

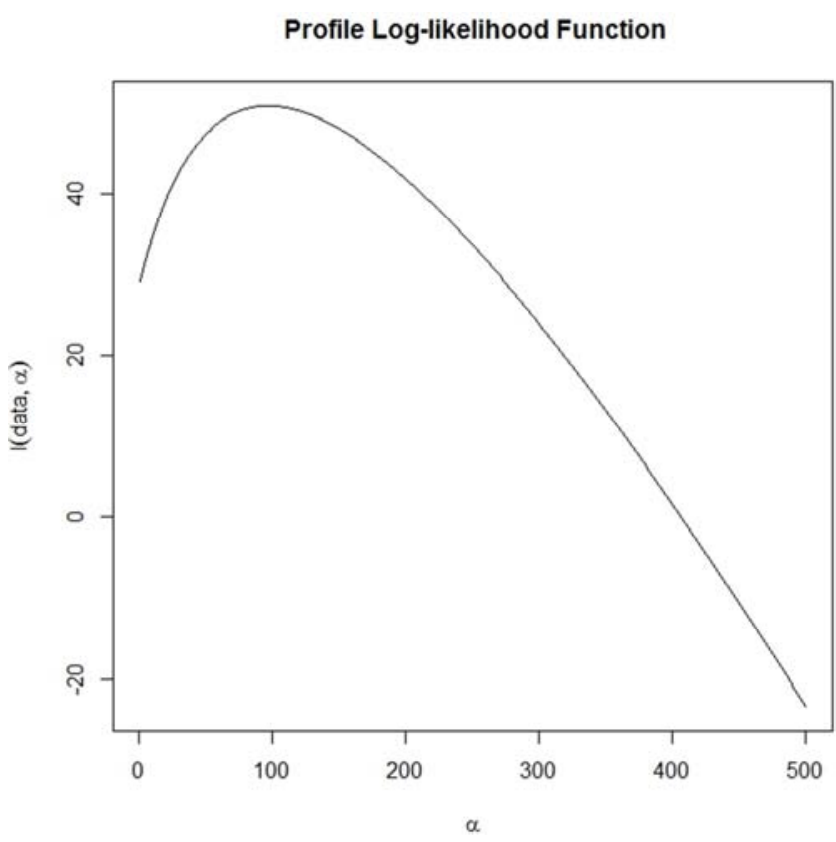

Figure 2. Profile Log-likelihood graph of a Gamma model. 


\subsection{Discussion}

By applying the Newton Raphson Method using simulation study, a total of 19 iterations were performed to obtain the maximum likelihood estimates of Gamma model. Convergence was achieved at the $17^{\text {th }}$ returning 509.3494 as the value of the log-likelihood and the values of the estimate which maximizes the function was 2.188828 and 7.959372 with gradient $-1.080348 \times 10^{-5}$ and $-6.056159 \times 10^{-6}$. The values of the hessian matrix were

$=\left[\begin{array}{cc}945.422 & -125.63178 \\ -125.63178 & 34.5435\end{array}\right]$ which is the second derivative.

Table 4 shows the estimates which maximizes the likelihood function of Gamma probability distribution for different sizes which ranges between (2.728430 and $2.728428)$ and (9.921558 and 9.9221553) with standard error reducing as the sample size increases $(1.15490$ to $0.03649552)$ and (4.607174 to 0.14569157$)$.

By applying the Newton Raphson method to get the Maximum likelihood estimates for the profile Gamma model likelihood function, a total of 10 iterations were performed to get the maximum likelihood estimate. Convergence was reached at the $10^{\text {th }}$ returning -50.76524 as the value of the log-likelihood and the value of the estimate which maximizes the function was 2.728441 with gradient $-11.1625181 \times 10^{-5}$. The variance was 2.515193 which is the second derivative.

Table 5 shows the estimate which maximizes the likelihood function of profile Gamma likelihood function for different sizes which is between (4.8621 and 5.040472) with standard error reducing as the sample size increases $(0.7082003$ to 0.02275701$)$ whereas in the case of real life data the iterations generated by applying Newton Raphson Method in obtaining maximum likelihood estimates for the Gamma model indicates a total of 31 iterations were performed to get the maximum likelihood estimates. Convergence was achieved at the $29^{\text {th }}$ returning 642.6958 as the value of the log-likelihood and the value of the estimate which maximizes the function was 0.850994171 and 0.001815127 with gradient $1.59319 \times 10-3$ and $4.028254 \times$ $10^{-6}$. The entries of the Hessian matrix were $=\left[\begin{array}{cc}188.0602 & -125.63178 \\ -48212.9634 & 20879859.92\end{array}\right]$ which the second derivative is.

Table 6 shows the estimates which maximizes the likelihood function of Gamma probability distribution $(0.851554121$ and 0.001817164$)$ with standard error of $(0.1141584591$ and 0.0003426042$)$. Similarly, by applying the Newton Raphson method to get the Maximum likelihood estimates for the profile Gamma model likelihood function, a total of 9 iterations were performed. Convergence was reached at the $8^{\text {th }}$ returning -5487.088 as the value of the loglikelihood and the value of the estimate which maximizes the function was 1.984034 with gradient $8.067959 \times 10^{-05}$. The variance was 3649.93 which was the second derivative. Table 7 shows the estimate which maximizes the likelihood function of profile Gamma likelihood function was (1.984034) with standard error of $(0.001655228)$.

\section{Conclusion}

Parameter estimates from the Gamma model can easily be obtained by the method of Maximum Likelihood Estimation jointly numerical approach (Newton Raphson method) with the help of computer. The result of the study shows that Maximum Likelihood Estimation (MLE) jointly with Newton Raphson method is more efficient for estimating parameters of the Gamma model on real life data than simulation study. Comparing Tables 4 and 5 it can be concluded that the estimate values of $\alpha$ that maximizes Gamma model and profile likelihood Gamma function are close to one another. Similarly, Comparing Tables 6 and 7 it can be concluded that the estimate values of $\alpha$ that maximizes gamma probability distribution and profile likelihood gamma are also not significantly different. It was observed that as the sizes of the sample increases the standard error reduces which obey the law of large numbers.

\section{Recommendation}

Based on the results drawn from this study; we recommend that the parameter estimates from the twoparameter Gamma model should be obtained using Maximum Likelihood Estimation jointly with Newton Raphson Method.

\section{References}

[1] Aldrich J.; R. A. Fisher and the Making of Maximum Likelihood 1912-1922, Statistical

[2] Atkinson, A. C., Pericchi, L. R., \& Smith, R. L. (1991). Grouped likelihood for the shifted power transformation. Journal of the Royal Statistical Society: Series B, 53: 473- 482.

[3] Beaumont, M. A., Zhang, W., \& Balding, D. J.(2002). Approximate Bayesian computation in population genetics. Genetics, 2 (162), 2025-2035.

[4] Cheng, R. C. H. \& Amin, N. A. K. (1983). Estimating parameters in continuous univariate distributions with a shifted origin. Journal of the Royal Statistical Society: Series $B, 45$ : 394-403.

[5] Cheng, R. C. H. \& Traylor, L. (1995). Non-regular maximum likelihood problems. Journal of the Royal Statistical Society: Series B, 57: 3-44.

[6] Cox, D. R. \& Reid, N. (2004). A note on pseudolikelihood constructed from marginal densities., Biometrika, 2( 91), 729-737.

[7] Cramer H.; Mathematical Methods of Statistics, Princeton University Press, 1946.

[8] Cule, M. L., Samworth, R. J. \& Stewart, M. I. (2010). Maximum likelihood estimation of a multi-dimensional logconcave density. Journal Royal Statistical Society B 72: 545600 .

[9] Dean, T. A., Singh, S. S., Jasra, A. \& Peters G. W. (2011). Parameter estimation for hidden Markov models with intractable likelihoods., Arxiv preprint ar Xiv:1103.5399v1. 
[10] Didelot, X., Everitt, R. G., Johansen, A. M. and Lawson, D. J. (2011). Likelihood-free estimation of model evidence. Bayesian Analysis, vol. 6,49-76.

[11] Fukunaga, K. \& Hostetler, L. D. (1975). The Estimation of the Gradient of a Density Function, with Applications in Pattern Recognition. IEEE Transactions on Information Theory 21: 3240 .

[12] Fearnhead, P. \& Prangle, D. (2012)."Constructing Summary Statistics for Approximate Bayesian Computation: Semiautomatic $\mathrm{ABC}$ (with discussion). Journal of the Royal Statistical Society Series B (Methodology) in press.

[13] Fisher, R. A. (1922). On the mathematical foundations of theoretical statistics.Philosophical Transactions of the Royal Society of London: Series A, 222: 309-368.

[14] Nemes G.; New asymptotic expansion for the $\Gamma(\mathrm{z})$ function, Stan's Library, Volume II, 2007.

[15] Marjoram, P., Molitor, J., Plagnol, V., \&Tavare, S. (2003). Markov chain Monte Carlo without likelihoods. Proceedings of the National Academy of Sciences USA: 1532415328.
[16] Pritchard, J. K., Seielstad, M. T., Perez-Lezaun, A., \& Feldman, M. T. (1999). Population Growth of Human Y Chromosomes: A Study of Y Chromosome Microsatellites. Molecular Biology and Evolution 16: 17911798.

[17] Pyke R.; Spacing, Journal of the Royal Statistical Statistical Society Series B, 27(3), 1965, pp. 395- 499.

[18] Robert, C. P., Cornuet, J., Marin, J. \& Pillai, N. S. (2011). Lack of confidence in $\mathrm{ABC}$ model choice. Proceedings of the National Academy of Sciences of the United States of America 108: 1511215117.

[19] Rockette H., Antle C., Klimko L. A.; Maximum likelihood model, Journal of the American Statistical Association, 69(345), 1974, pp. 246-249.

[20] Rosin and Rammler (1933) application of Weibull distribution to describe the size distribution of particles

[21] Wilkinson, R. D. (2008). Approximate Bayesian computation $(\mathrm{ABC})$ gives exact results under the assumption of error model. Arxiv preprint ar Xiv:0811.3355. 